Proceedings of the Institute of Mathematics and Mechanics,

National Academy of Sciences of Azerbaijan

Volume 46, Number 2, 2020, Pages 189-196

https://doi.org/10.29228/proc.28

\title{
SOME PROPERTIES OF THE SPECTRUM OF THE DIRAC OPERATOR WITH A SPECTRAL PARAMETER IN THE BOUNDARY CONDITION
}

\author{
ABID G. FERZULLAZADEH AND IBRAHIM M. NABIEV
}

\begin{abstract}
In this paper, we consider the Dirac operator subject to nonseparated boundary conditions of which one contains the spectral parameter. The representations of some entire functions are given, the reality eigenvalues and the absence of associated vector functions to the vector eigenfunctions are proved, an asymptotic formula for the eigenvalues of the Dirac operator are obtained.
\end{abstract}

\section{Introduction}

The one-dimensional stationary Dirac system (related to the behavior of a relativistic electron in an electrostatic field) has the following canonical form:

$$
B Y^{\prime}(x)+Q(x) Y(x)=\lambda Y(x),
$$

where $\lambda$ is the spectral parameter, $B=\left(\begin{array}{cc}0 & 1 \\ -1 & 0\end{array}\right), Q(x)=\left(\begin{array}{cc}p(x) & q(x) \\ q(x) & -p(x)\end{array}\right)$, $Y(x)=\left(\begin{array}{l}y_{1}(x) \\ y_{2}(x)\end{array}\right)$

Suppose that the elements $p(x)$ and $q(x)$ matrices $Q(x)$ in (1.1) are real functions belonging to the space $W_{2}^{1}[0, \pi]$. By $W_{2}^{1}[0, \pi]$ we denote the space consisting of absolutely continuous functions defined on a segment $[0, \pi]$, which have a derivative, summable with a square on $[0, \pi]$. Consider a boundary problem generated on a segment $[0, \pi]$ by the Dirac equation (1.1) and the boundary conditions of the form

$$
\begin{aligned}
& (\alpha \lambda+\beta) y_{1}(0)+y_{2}(0)+\omega y_{1}(\pi)=0, \\
& -\bar{\omega} y_{1}(0)+\gamma y_{1}(\pi)+y_{2}(\pi)=0,
\end{aligned}
$$

where $\alpha, \beta, \gamma$ are real numbers, $\omega$ is a complex number, and $\alpha \omega \neq 0$. The boundary-value problem (1.1), (1.2) will be denoted by $D$.

At present, many questions of direct and inverse problems for Dirac operators in the case of separated and non-separated boundary conditions are well studied. Thus, direct problems for such operators were studied in $[5,9,11,12,21]$, and questions on the recovery of operators - in $[1,2,3,4,6,7,8,10,13,14,15,17,18$,

2010 Mathematics Subject Classification. Primary 34A55, 34B24; Secondary 47E05.

Key words and phrases. Dirac operator, non-separated boundary conditions, asymptotics of the eigenvalues. 
$19,20,23]$ and other works. In particular, in papers $[3,4,6,7,8,13,14,15,23]$ a number of important results were obtained in the theory of inverse problems for systems of differential equations under separated boundary conditions. [1, 2, 10, $17,18,19,20]$ are devoted to the study of inverse spectral problems for equation (1.1) with various types of nonseparated boundary conditions, including periodic, antiperiodic, and quasiperiodic $[10,17,19,20]$ boundary conditions.

In this paper, we consider the Dirac operator subject to non-separated boundary conditions of which one contains the spectral parameter. The representations of some entire functions are given, the reality eigenvalues and the absence of associated vector functions to the vector eigenfunctions are proved, an asymptotic formula for the eigenvalues of the boundary value problem $D$ are obtained.

\section{Some spectral properties of the boundary value problem}

Denote by $C(x, \lambda)=\left(\begin{array}{c}c_{1}(x, \lambda) \\ c_{2}(x, \lambda)\end{array}\right)$ and $S(x, \lambda)=\left(\begin{array}{c}s_{1}(x, \lambda) \\ s_{2}(x, \lambda)\end{array}\right)$ are solutions of equation (1.1) satisfying the initial conditions

$$
C(0, \lambda)=\left(\begin{array}{l}
1 \\
0
\end{array}\right), S(0, \lambda)=\left(\begin{array}{l}
0 \\
1
\end{array}\right) .
$$

Lemma 2.1. The following representations hold:

$$
\begin{gathered}
c_{1}(\pi, \lambda)=\cos \lambda \pi+\psi_{1}(\lambda), c_{2}(\pi, \lambda)=\sin \lambda \pi+\psi_{2}(\lambda), \\
s_{1}(\pi, \lambda)=-\sin \lambda \pi+A_{1} \frac{\cos \lambda \pi}{\lambda}+B_{1} \frac{\sin \lambda \pi}{\lambda}+\frac{\psi_{3}(\lambda)}{\lambda}, \\
s_{2}(\pi, \lambda)=\cos \lambda \pi+A_{2} \frac{\sin \lambda \pi}{\lambda}+B_{2} \frac{\cos \lambda \pi}{\lambda}+\frac{\psi_{4}(\lambda)}{\lambda},
\end{gathered}
$$

where

$$
\begin{gathered}
A_{1}=A+Q_{1}, A_{2}=A+Q_{2}, B_{1}=-\frac{p(0)+p(\pi)}{2}, B_{2}=\frac{p(0)-p(\pi)}{2} \\
A=\frac{1}{2} \int_{0}^{\pi}\left[p^{2}(x)+q^{2}(x)\right] d x, Q_{1}=\frac{q(\pi)-q(0)}{2}, Q_{2}=-\frac{q(0)+q(\pi)}{2}, \\
\psi_{j}(\lambda)=\int_{-\pi}^{\pi} \tilde{\psi}_{j}(t) e^{i \lambda t} d t, \tilde{\psi}_{j}(t) \in L_{2}[-\pi, \pi], j=\overline{1,4}
\end{gathered}
$$

The proof of the lemma follows from [16, p. 66].

Definition 2.1. A complex number $\lambda_{0}$ is called an eigenvalue of boundary value problem $D$ if equation (1.1) with $\lambda=\lambda_{0}$ has a nontrivial solution $Y_{0}(x)$ satisfying the boundary conditions (1.2); in this case the vector function $Y_{0}(x)$ is called the vector eigenfunction of problem $D$ corresponding to the eigenvalue $\lambda_{0}$. The set of eigenvalues is called the spectrum of $D$. The vector functions

$$
Y_{1}(x)=\left(\begin{array}{l}
y_{1,1}(x) \\
y_{2,1}(x)
\end{array}\right), Y_{2}(x)=\left(\begin{array}{l}
y_{1,2}(x) \\
y_{2,2}(x)
\end{array}\right), \ldots, Y_{r}(x)=\left(\begin{array}{l}
y_{1, r}(x) \\
y_{2, r}(x)
\end{array}\right)
$$


are called as the associated vector functions to the vector eigenfunction $Y_{0}(x)=$ $\left(\begin{array}{l}y_{1,0}(x) \\ y_{2,0}(x)\end{array}\right)$, if they are absolutely continuous, they satisfy the differential equations

$$
B Y_{j}^{\prime}(x)+Q(x) Y_{j}(x)-Y_{j-1}(x)=\lambda_{0} Y_{j}(x)
$$

and boundary conditions

$$
\begin{gathered}
(\alpha \lambda+\beta) y_{1, j}(0)+y_{2, j}(0)+\omega y_{1, j}(\pi)+\alpha y_{1, j-1}(0)=0 \\
-\bar{\omega} y_{1, j}(0)+\gamma y_{1, j}(\pi)+y_{2, j}(\pi)=0 \\
j=1,2,3, \ldots, r .
\end{gathered}
$$

Theorem 2.1. When $\alpha<0$ the eigenvalues of the problem $D$ are real.

Proof. Let $\lambda$ be an eigenvalue of the boundary value problem $D$, and let $Y(x)=$ $\left(\begin{array}{l}y_{1}(x) \\ y_{2}(x)\end{array}\right)$ be the corresponding vector eigenfunction. Multiply the equality (1.1) by $\left(\overline{y_{1}(x)}, \overline{y_{2}(x)}\right)$ on the left,

$$
B \overline{Y^{\prime}(x)}+Q(x) \overline{Y(x)}=\bar{\lambda} \overline{Y(x)}
$$

by $\left(y_{1}(x), y_{2}(x)\right)$ and subtract one product from the other. As a result, we get

$$
(\lambda-\bar{\lambda})\left[\left|y_{1}(x)\right|^{2}+\left|y_{2}(x)\right|^{2}\right]=\frac{d}{d x}\left[\overline{y_{1}(x)} y_{2}(x)-y_{1}(x) \overline{y_{2}(x)}\right] .
$$

Integrating this equality from zero to $\pi$, we find

$$
\begin{gathered}
2 i \operatorname{Im} \lambda \int_{0}^{\pi}\left[\left|y_{1}(x)\right|^{2}+\left|y_{2}(x)\right|^{2}\right] d x= \\
=\overline{y_{1}(\pi)} y_{2}(\pi)-y_{1}(\pi) \overline{y_{2}(\pi)}-\overline{y_{1}(0)} y_{2}(0)+y_{1}(0) \overline{y_{2}(0)} .
\end{gathered}
$$

It follows from the boundary conditions (1.2) that

$$
\begin{aligned}
& y_{2}(0)=-\omega y_{1}(\pi)-(\alpha \lambda+\beta) y_{1}(0) \\
& y_{2}(\pi)=\bar{\omega} y_{1}(0)-\gamma y_{1}(\pi) .
\end{aligned}
$$

Let's consider these at the equality (2.4):

$$
\begin{gathered}
2 i \operatorname{Im} \lambda \int_{0}^{\pi}\left[\left|y_{1}(x)\right|^{2}+\left|y_{2}(x)\right|^{2}\right] d x= \\
=\overline{y_{1}(\pi)}\left[\bar{\omega} y_{1}(0)-\gamma y_{1}(\pi)\right]-y_{1}(\pi)\left[\omega \overline{y_{1}(0)}-\gamma \overline{y_{1}(\pi)}\right]+ \\
+\overline{y_{1}(0)}\left[\omega y_{1}(\pi)+(\alpha \lambda+\beta) y_{1}(0)\right]-y_{1}(0)\left[\bar{\omega} \overline{y_{1}(\pi)}+(\alpha \bar{\lambda}+\beta) \overline{y_{1}(0)}\right]= \\
=2 i \alpha\left|y_{1}(0)\right|^{2} \operatorname{Im} \lambda .
\end{gathered}
$$

Consequently,

$$
\operatorname{Im} \lambda\left\{\int_{0}^{\pi}\left[\left|y_{1}(x)\right|^{2}+\left|y_{2}(x)\right|^{2}\right] d x-\alpha\left|y_{1}(0)\right|^{2}\right\}=0 .
$$

Since $\alpha<0$, the expression in braces is distinct from zero. Therefore, $\operatorname{Im} \lambda=0$.

Theorem 2.1 is proved.

Theorem 2.2. If $\alpha<0$, then the vector eigenfunctions of the boundary value problem $D$ have no associated vector functions. 
Proof. Let's assume the opposite. Suppose that, the boundary value problem $D$ has an vector eigenfunction of $Y_{1}(x)=\left(\begin{array}{l}y_{1,1}(x) \\ y_{2,1}(x)\end{array}\right)$ associated to the $Y_{0}(x)=$ $\left(\begin{array}{l}y_{1,0}(x) \\ y_{2,0}(x)\end{array}\right)$ vector eigenfunction, corresponding to the eigenvalue $\lambda_{0}$. Then, by virtue of (1.1), (2.2), the following equalities hold:

$$
\begin{gathered}
B \overline{Y_{0}^{\prime}(x)}+Q(x) \overline{Y_{0}(x)}=\lambda_{0} \overline{Y_{0}(x)}, \\
B Y_{1}^{\prime}(x)+Q(x) Y_{1}(x)-Y_{0}(x)=\lambda_{0} Y_{1}(x) .
\end{gathered}
$$

Multiply the equality $(2.5)$ by $\left(y_{1,1}(x), y_{2,1}(x)\right)$ - from the left and equality (2.6) by $\left(\overline{y_{1,0}(x)}, \overline{y_{2,0}(x)}\right)$ and subtract them side by side, we will get

$$
\left|y_{1,0}(x)\right|^{2}+\left|y_{2,0}(x)\right|^{2}=\frac{d}{d x}\left[y_{2,1}(x) \overline{y_{1,0}(x)}-y_{1,1}(x) \overline{y_{2,0}(x)}\right] .
$$

Integrating this equality from zero to $\pi$ for $x$, we obtain

$$
\begin{gathered}
\int_{0}^{\pi}\left[\left|y_{1,0}(x)\right|^{2}+\left|y_{2,0}(x)\right|^{2}\right] d x= \\
=y_{2,1}(\pi) \overline{y_{1,0}(\pi)}-y_{1,1}(\pi) \overline{y_{2,0}(\pi)}-y_{2,1}(0) \overline{y_{1,0}(0)}+y_{1,1}(0) \overline{y_{2,0}(0)} .
\end{gathered}
$$

According to the boundary conditions (1.2) and (2.3), we have

$$
\begin{gathered}
y_{2,0}(0)=-\omega y_{1,0}(\pi)-(\alpha \lambda+\beta) y_{1,0}(0), \\
y_{2,0}(\pi)=\bar{\omega} y_{1,0}(0)-\gamma y_{1,0}(\pi), \\
y_{2,1}(0)=-(\alpha \lambda+\beta) y_{1,1}(0)-\omega y_{1,1}(\pi)-\alpha y_{1,0}(0), \\
y_{2,1}(\pi)=\bar{\omega} y_{1,1}(0)-\gamma y_{1,1}(\pi) .
\end{gathered}
$$

By substituting these expressions into (2.7), we find

$$
\begin{gathered}
\int_{0}^{\pi}\left[\left|y_{1,0}(x)\right|^{2}+\left|y_{2,0}(x)\right|^{2}\right] d x= \\
=\overline{y_{1,0}(\pi)}\left[\bar{\omega} y_{1,1}(0)-\gamma y_{1,1}(\pi)\right]-y_{1,1}(\pi)\left[\omega \overline{y_{1,0}(0)}-\gamma \overline{y_{1,0}(\pi)}\right]+ \\
+\overline{y_{1,0}(0)}\left[(\alpha \lambda+\beta) y_{1,1}(0)+\omega y_{1,1}(\pi)+\alpha y_{1,0}(0)\right]- \\
-y_{1,1}(0)\left[\bar{\omega} \overline{y_{1,0}(\pi)}+(\alpha \lambda+\beta) \overline{y_{1,0}(0)}\right]=\alpha\left|y_{1,0}(0)\right|^{2} .
\end{gathered}
$$

Hence

$$
\int_{0}^{\pi}\left[\left|y_{1,0}(x)\right|^{2}+\left|y_{2,0}(x)\right|^{2}\right] d x-\alpha\left|y_{1,0}(0)\right|^{2}=0 .
$$

This contradicts the fact that the left side of this relation is positive according to the inequality $\alpha<0$. Theorem 2.2 is proved. 


\section{Asymptotics of eigenvalues}

Theorem 3.1. The boundary value problem $D$ has a countable set of eigenvalues $\gamma_{k}(k= \pm 0, \pm 1, \pm 2, \ldots)$. These eigenvalues satisfy the asymptotic formula

$$
\begin{gathered}
\gamma_{k}=k+a+\frac{A}{\pi k}+ \\
+\frac{4(-1)^{k} b \operatorname{Re} \omega+\alpha q(\pi)\left(\gamma^{2}-1\right)-\alpha b^{2} q(0)-2 \alpha \gamma p(\pi)-2 b^{2}-2|\omega|^{2}}{2 \pi \alpha b^{2} k}+\frac{\xi_{k}}{k},
\end{gathered}
$$

as $|k| \rightarrow \infty$, where $a=\frac{1}{\pi} \operatorname{arcctg} \gamma, \quad b=\sqrt{1+\gamma^{2}},\left\{\xi_{k}\right\} \in l_{2}$.

Proof. The general solution of the equation (1.1) has a form

$$
y(x, \lambda)=M_{1} S(x, \lambda)+M_{2} C(x, \lambda),
$$

where $M_{1}, M_{2}$ are arbitrary constants. Considering the boundary conditions (1.2), the initial conditions (2.1) and taking into account the identity

$$
c_{1}(x, \lambda)\left[s_{2}(x, \lambda)+\gamma s_{1}(x, \lambda)\right]-s_{1}(x, \lambda)\left[c_{2}(x, \lambda)+\gamma c_{1}(x, \lambda)\right]=1
$$

it is easy to verify that the characteristic function of the boundary value problem $D$ will be

$$
d(\lambda)=2 \operatorname{Re} \omega-\varphi(\lambda)+|\omega|^{2} s_{1}(\pi, \lambda)+(\alpha \lambda+\beta) \theta(\lambda),
$$

where

$$
\varphi(\lambda)=c_{2}(\pi, \lambda)+\gamma c_{1}(\pi, \lambda), \theta(\lambda)=s_{2}(\pi, \lambda)+\gamma s_{1}(\pi, \lambda) .
$$

The zeros of the function $d(\lambda)$ are the eigenvalues of the problem $D$.

Using the representations in the lemma 2.1, we transform the characteristic function (3.2) to the form

$$
\begin{aligned}
d(\lambda)= & 2 \operatorname{Re} \omega+\alpha \lambda(\cos \lambda \pi-\gamma \sin \lambda \pi)+\left(\alpha \gamma A_{1}+\alpha B_{2}+\beta-\gamma\right) \cos \lambda \pi+ \\
& +\left(\alpha A_{2}+\alpha \gamma B_{1}-1-|\omega|^{2}-\beta \gamma\right) \sin \lambda \pi+\psi_{5}(\lambda),
\end{aligned}
$$

where $\psi_{5}(\lambda)=\int_{-\pi}^{\pi} \tilde{\psi}_{5}(t) e^{i \lambda t} d t, \tilde{\psi}_{5}(t) \in L_{2}[-\pi, \pi]$.

Let denote the contour bounded a square

$$
K_{n}=\left\{\lambda:|\operatorname{Re} \lambda-a| \leq n+\frac{1}{2},|\operatorname{Im} \lambda| \leq n+\frac{1}{2}\right\}
$$

by $\Gamma_{n}$. According to the relation (3.3), we have

$$
d(\lambda)=F(\lambda)+G(\lambda)
$$

where

$$
\begin{gathered}
F(\lambda)=\alpha \lambda(\cos \lambda \pi-\gamma \sin \lambda \pi), \\
G(\lambda)=2 \operatorname{Re} \omega+\left(\alpha \gamma A_{1}+\alpha B_{2}+\beta-\gamma\right) \cos \lambda \pi+ \\
+\left(\alpha A_{2}+\alpha \gamma B_{1}-1-|\omega|^{2}-\beta \gamma\right) \sin \lambda \pi+\psi_{5}(\lambda) .
\end{gathered}
$$

By the standart method (see, for example, [16, p. 43]), it can be shown that for sufficiently large $n$ on $\Gamma_{n}$, the inequality $|F(\lambda)|>|G(\lambda)|$ holds. Then, by Rouche's therom [22, p. 263], the square of $K_{n}$ contains the same number of zeros of the functions of $d(\lambda)$ and $F(\lambda)$, i.e. $2 n+2$. Denote these zeros in non-decreasing order of their modules by

$$
\gamma_{-n}, \gamma_{-n+1}, \ldots, \gamma_{-1}, \gamma_{-0}, \gamma_{+0}, \gamma_{1}, \ldots, \gamma_{n-1}, \gamma_{n}
$$


Hence, the problem $D$ has a counting number of eigenvalue.

Applying Rouché's theorem, it is easy to verify that the zeros of function (3.3) when $k \rightarrow \pm \infty$ obey the asymptotic formula

$$
\gamma_{k}=k+a+\varepsilon_{k},
$$

where $\varepsilon_{k}=O\left(k^{-1}\right)$. Let's precise the asymptotic of number $\varepsilon_{k}$. We will obtain the following equalities if we use from the separation of the functions $\sin x$ and $\cos x$ for this.

$$
\begin{gathered}
\cos \gamma_{k} \pi=(-1)^{k} \cos \left(a+\varepsilon_{k}\right) \pi=\frac{(-1)^{k} \gamma}{\sqrt{1+\gamma^{2}}}-\frac{(-1)^{k}}{\sqrt{1+\gamma^{2}}} \varepsilon_{k} \pi+O\left(\frac{1}{k^{2}}\right), \\
\sin \gamma_{k} \pi=(-1)^{k} \sin \left(a+\varepsilon_{k}\right) \pi=(-1)^{k}\left(\frac{1}{\sqrt{1+\gamma^{2}}}+\frac{\gamma}{\sqrt{1+\gamma^{2}}} \varepsilon_{k} \pi\right)+O\left(\frac{1}{k^{2}}\right) .
\end{gathered}
$$

Then, we obtain,

$$
\begin{gathered}
\frac{2 \operatorname{Re} \omega}{\gamma_{k}}=\frac{2 \operatorname{Re} \omega}{k}+o\left(\frac{1}{k^{2}}\right), \\
\frac{\cos \gamma_{k} \pi}{\gamma_{k}}=(-1)^{k} \frac{\gamma}{\sqrt{1+\gamma^{2}}} \cdot \frac{1}{k}+o\left(\frac{1}{k^{2}}\right), \\
\frac{\sin \gamma_{k} \pi}{\gamma_{k}}=\frac{(-1)^{k}}{\sqrt{1+\gamma^{2}}} \cdot \frac{1}{k}+o\left(\frac{1}{k^{2}}\right), \\
\frac{\psi_{5}\left(\gamma_{k}\right)}{\gamma_{k}}=\frac{\tau_{k}}{k},\left\{\tau_{k}\right\} \in l_{2} .
\end{gathered}
$$

By plugging this expression into the equation $d(\lambda)=0$, we obtain the asymptotic formula

$$
\begin{gathered}
\frac{2 \operatorname{Re} \omega}{k}+\alpha\left(\frac{(-1)^{2} \gamma}{\sqrt{1+\gamma^{2}}}-\frac{(-1)^{k}}{\sqrt{1+\gamma^{2}}} \varepsilon_{k} \pi-\gamma\left(\frac{(-1)^{k}}{\sqrt{1+\gamma^{2}}}+\frac{(-1)^{k} \gamma}{\sqrt{1+\gamma^{2}}} \varepsilon_{k} \pi\right)\right)+ \\
+\left(\alpha \gamma A_{1}+\alpha B_{2}-\gamma+\beta\right) \cdot \frac{(-1)^{k} \gamma}{k \sqrt{1+\gamma^{2}}}+ \\
+\left(\alpha A_{2}-1-|\omega|^{2}+\alpha \gamma B_{1}-\beta \gamma\right) \cdot \frac{(-1)^{k}}{k \sqrt{1+\gamma^{2}}}+\frac{\eta_{k}}{k}=0,\left\{\eta_{k}\right\} \in l_{2} .
\end{gathered}
$$

Then,

$$
\varepsilon_{k}=\frac{4(-1)^{k} b \operatorname{Re} \omega+\alpha q(\pi)\left(\gamma^{2}-1\right)-\alpha b^{2} q(0)-2 \alpha \gamma p(\pi)-2 b^{2}-2|\omega|^{2}}{2 \pi \alpha b^{2} k}+\frac{\xi_{k}}{k},
$$

Taking into account this formula in expression (3.4), we obtain the desired formula (3.1). Theorem 3.1 is proved.

\section{Acknowledgements}

This work was supported by the Science Development Foundation under the President of the Republic of Azerbaijan -Grant No EIF/MQM/Elm-Tehsil-12016-1(26)-71/05/1. 


\section{References}

[1] N.V. Abbasli, I.M. Nabiev, The uniqueness of the reconstruction of the Dirac system by three spectra, J. Contemporary Appl. Math. 8 (2018), 3-8.

[2] T.Sh. Abdullaev, I.M. Nabiev, An Algorithm for Reconstructing the Dirac Operator with a Spectral Parameter in the Boundary Condition, Comput. Math. Math. Phys. 56 (2016), no.2, 256-262.

[3] S. Albeverio, R. Hryniv, Y. Mykytyuk, Inverse spectral problemsfor Dirac operators with summable potentials, Russian J.Math. Phys. 12 (2005), no.4, 406-423.

[4] R. Kh. Amirov, B. Keskin, A. S. Ozkan, Direct and inverse problems for the Dirac operator with spectral parameter linearly contained in boundary condition, Ukrainian Math. J. 61 (2009), no.9, 1365-1379.

[5] S. Currie, T.T. Roth, B.A. Watson, Eigenvalue interlacing for first order differential systems with periodic $2 \times 2$ matrix potentials and quasi-periodic boundary conditions, Operators and Matrices 12 (2018), no.2, 489-499.

[6] M.G. Gasymov, T.T. Dzhabiev, Determination of a system of Dirac differential equations using two spectra, in: Proceedings of School-Seminar on the Spectral Theory of Operators and Representations of Group Theory, Baku, Elm, 1975, 46-71 (in Russian).

[7] O. Gorbunov, V. Yurko, Inverse problem for Dirac system with singularities in interior points, Anal. Math. Phys. 6 (2016), no.1, 1-29.

[8] Y. Guo, G. Wei, R. Yao, Inverse problems for Dirac operator with the potential known on an interior subinterval, Anal. Math. Phys. 9 (2019), no.1, 155-163.

[9] H.M. Huseynov, A.R. Latifova, On eigenvalues and eigenfunctions of one class of Dirac operators with discontinuous coefficients, Trans. Natl. Acad. Sci. Azerb. 24 (2004), no.1, 103-112.

[10] E. Korotyaev, Inverse problem and estimates for periodic Zakharov-Shabat systems, J. Reine Angew. Math. 583 (2005), 87-115.

[11] V.M. Kurbanov, A.M. Abdullayeva, Bessel property and basicity of the system of root vector-functions of Dirac operator with summable coefficient, Operators and Matrices. 12 (2018), no.4, 943-954.

[12] A.A. Lunyov, M. M. Malamud, On the Riesz basis property of root vectors system for $2 \times 2$ Dirac type operators, J. Math. Anal. Appl. 441 (2016), 57-103.

[13] Kh.R. Mamedov, O. Akcay, Inverse eigenvalue problem for a class of Dirac operators with discontinuous Coefficient, Boundary Value Problems 2014 (2014), 1-20.

[14] Kh.R. Mamedov, O. Akcay, Inverse problem for a class of Dirac operators by the Weyl function, Dynamic Systems and Appl. 26 (2017), 183 -196.

[15] Kh.R. Mamedov, O. Akcay, Inverse problem for a class of Dirac operator, Taiwanese J. Math. 13 (2014), no.3, 753-772.

[16] V.A. Marchenko, Sturm-Liouville operators and their applications, Naukova Dumka, Kiev, 1977 (in Russian).

[17] T. V. Misyura, A characteristic of the spectra of periodic and antiperiodic boundary value problems for the Dirac operator. II, Teor. Funk. Funk. Anal. Prilozh. (1979), no.31, 102-109.

[18] I.M. Nabiev, Solution of a class of inverse problems for the Dirac operator, Trans. Acad. sci. Azerb., ser. phys.-tech. math. sci. 21 (2001), no.1, 146-157.

[19] I.M. Nabiev, Solution of the inverse quasi-periodic problem for the Dirac system, Math. Notes 89 (2011), no.6, 845-852.

[20] I.M. Nabiev, The inverse periodic problem for the Dirac operator,Proc. IMM of NAS of Azerb. 19 (2003), 177-180.

[21] A.M. Savchuk, A. A. Shkalikov, Dirac operator with complex-valued summable potential, Math. Notes 96 (2014), 777-810. 
[22] Yu.V. Sidorov, M.V. Fedoryuk, M.I. Shabunin, Lectures on the theory of functions of a complex variable, Nauka, Moscow, 1982 (in Russian).

[23] Ch.-Fu Yang, D.-Q. Liu, Half-inverse problem for the Dirac operator, Appl. Math. Letters 87 (2019), 172-178.

Abid G. Ferzullazadeh

Lankaran State University, General Hazi Aslanov 50, Lankaran AZ4200, Azerbaijan

E-mail address: abid.ferzullazade@mail.ru

Ibrahim M. Nabiev

Baku State University, Z. Khalilov 23, Baku AZ1148, Azerbaijan

Institute of Mathematics and Mechanics, National Academy of Sciences of Azerbaijan, B. Vahabzadeh 9, Baku AZ1141, Azerbaijan

Khazar University, Mahsati 11, Baku AZ1096, Azerbaijan

E-mail address: nabievim@yahoo.com

Received: March 14, 2020; Revised: May 26, 2020; Accepted: June 16, 2020 